\title{
PORQUE SÍ NOS IMPORTA. LA IMPLICACIÓN EDUCATIVA DE LAS MADRES MARROQUÍES INMIGRANTES EN CATALUÑ ${ }^{1}$
}

\author{
Rosalina Alcalde Campos \\ Universidad Autónoma de Barcelona \\ Judith Martos Martín \\ Graduada en Educación Infantil, Universidad Autónoma de Barcelona
}

\begin{abstract}
RESUMEN: Las conductas de implicación educativa de padres y madres pueden explicar en gran medida los resultados académicos de los descendientes, según demuestran diversidad de investigaciones en el campo de la psicología y de la sociología. Esto abarca también a las familias de origen inmigrante. Desde la sociología de la educación, numerosos estudios han confirmado que las hijas e hijos de padres inmigrantes obtienen peores resultados académicos. Si bien en España los estudios que analizan en profundidad las conductas de implicación parental de estas familias como factor explicativo no son abundantes. En el caso español, sabemos que el colectivo marroquí presenta un rendimiento escolar por debajo de los estudiantes españoles pero también respecto a otros grupos de alumnos de origen inmigrante. Por ello este artículo examina hasta qué punto la implicación parental podría estar involucrada en este fenómeno. Nuestro trabajo visibiliza también el protagonismo que las mujeres madres marroquíes tienen en estos procesos, acorde a una perspectiva de género femenino. Los resultados de nuestro estudio cualitativo, con 14 madres de origen marroquí residentes en Barcelona, indican que el seguimiento escolar que realizan es considerablemente alto. Incluso en situaciones de riesgo de exclusión social y de condiciones de pobreza.
\end{abstract}

PALABRAS CLAVE: Implicación parental, inmigración marroquí, expectativas educativas, socialización familiar, España, Barcelona.

\section{BECAUSE WE ARE CONCERN: THE PARENTAL INVOLVEMENT OF MOROCCAN IMMIGRANT MOTHERS IN CATALONIAN}

ABSTRACT: As numerous studies demonstrate the parental involvement can explain in a high part the academic outputs of children. Also among immigrant

1. En esta investigación participaron también Desiré Zabala, Anabel García, Mari Ángeles Fuerte, Berta Lajara, Nerea Martínez. 
families. From sociology of education, many studies have confirmed that immigrant children have worse academic achievement. However, in Spain, far too little attention has been paid to parental involvement in immigrant families as an element correlates with school achievement. In the Spanish case, we know that the Moroccan students have a school performance below other students. The aim of this paper is to examine if the parental involvement might be involved in the Moroccan children school achievement in 14 family cases. In particular, we show the mother's role in this process. The results obtained from this preliminary study indicate that parental involvement in these families is high. However, parental support in activities oriented to self-regulation is weak. And this element has a high correlation with school achievement.

KEYWORDS: Parental involvement, Moroccan immigrants, educative expectations, family socialization, Spain, Barcelona.

Recibido: 27/06/2016

Aceptado: 27/09/2016

Correspondencia: Rosalina Alcalde Campos, Universidad Autónoma de Barcelona, Departamento de Sociología, Avenida Eix Central, Edificio B, 08193 Bellaterra (Cerdanyola del Vallès) Barcelona. Email: rosalina.alcalde@uab.cat.

\section{INTRODUCCIÓN}

Las familias de origen inmigrante presentan particularidades propias del proceso migratorio y de su origen social que pueden conllevar ciertos obstáculos educativos en la experiencia escolar de sus hijas e hijos. Son muchos los estudios realizados sobre este tema que así los demuestran (Appelbaum, 2002; Aparicio y Tornos 2006; Carbonell, 2000; Elosegui y Etxeberría, 2010; Suárez-Orozco y Suárez-Orozco, 2008). No obstante, en España, los trabajos que expliquen la relación entre estrategias familiares de implicación y el rendimiento académico son poco abundantes. En este sentido consideramos que nuestra investigación cualitativa puede aportar elementos novedosos a la investigación en nuestro país. De manera que contribuya a comprender y actuar mejor sobre los contextos sociofamiliares de las familias migrantes de origen marroquí.

Por otra parte nos parece fundamental reconocer a las mujeres y madres marroquíes inmigrantes como figuras centrales que articulan tanto las dimensiones relacionales en el interior de la familia. Desde una perspectiva feminista del estudio de las migraciones y de los procesos educativos, nos parece necesario revalorizar el espacio familiar en la sociedad contemporánea. En este sentido, estimamos necesario dar a conocer que los modelos de socialización familiar que siguen estas familias convierten a estas mujeres en motores del rendimiento escolar de sus hijas e hijas. Trabajos como los de Vicente Llorent y $M^{a}$ Teresa Terrón (2013) sobre la inmigración femenina marroquí en España, destacan la importancia entre las familias marroquíes tanto de su modelo parental (entendido como modelo de socialización familiar) como de su identidad cultural. A partir de estos patrones, se trasmiten valores, jerarquías, normas y ex- 
pectativas de futuro que inciden en los patrones de conducta de estas madres a la hora de educar a sus hijas e hijos. Son las mujeres madres marroquíes las que poseen un rol principal en estos procesos de socialización familiar. Cuando las mujeres marroquíes se casan pasan a tener un papel clave en la sociedad como esposas y madres. Esto las convierte en una pieza central de la institución familiar por lo que una de sus principales funciones es la educación de los descendientes. Los padres se muestran ausentes en este proceso a causa de las responsabilidades laborales. Las madres se encargan de realizar el seguimiento y el control de los estudios así como de vigilar el entorno de los pequeños y establecer también la relación con la escuela (Terrón, 2008). Esto responde a la clásica división sexual del trabajo propia de los órdenes patriarcales.

Desde la sociología de la familia se han estudiado los modelos de socialización familiar considerando la combinación de los niveles de afecto y comunicación entre madres e hijas e hijos, junto con el nivel de control de la norma social. Esto da lugar a cuatro modelos de socialización familiar (Musitu, 2001): Contractual, también conocido como Conductivista o Autorizativo (Alto control sobre la norma y alto nivel afectivo y comunicativo); Permisivo o Negligente (bajo control de la norma y alto nivel afectivo y comunicativo); Autoritario (Alto control y baja comunicación y afectividad) y Anómico (Bajo control y baja afectividad y comunicación).

Los estudios sobre la materia han probado que el modelo que más correlaciona con el buen rendimiento escolar es el contractual-conductivista (Kellerhals y Montandon,1991; Collet y Tort, 2003). Estos modelos favorecen la implicación parental dado que proporcionan una distancia padres-hijos reducida, mucha comunicación con la niña y el niño, el fomento del diálogo íntimo y de la expresividad. Además, estos estilos parentales otorgan mucha importancia a la autorregulación y motivación del menor así como un alto control del autocumplimiento de las normas abiertas a la renegociación.

La implicación parental se refiere a los métodos que realizan las familias hacia el aprendizaje y rendimiento de sus hijas e hijos. Es decir, las formas específicas en que la conducta de la familia -para nuestro caso la conducta de las madres- incide sobre los procesos de aprendizaje y el rendimiento académico. La sociología de la educación ha demostrado ampliamente la relación existente entre la familia y el rendimiento escolar. Ruiz de Miguel (2001) hace una excelente revisión de los principales trabajos en este campo que indican que ciertas características del medio familiar dan lugar a un clima educativo y afectivo más o menos estimulante y motivador. El trabajo de Marjoribanks (1979) también señala que aspectos como la orientación intelectual, la presión para el logro y la aprobación parental parecen relacionarse con la inteligencia, el logro académico y otras características afectivas tales como el autoconcepto académico, el grado de ajuste escolar y la motivación de logro.

Sobre el origen social y los capitales culturales de las familias, las investigaciones del campo de la sociología de la educación han convenido que existe una relación positiva entre capitales culturales -fundamentalmente el de las madres- y la implicación parental (Martínez y Álvarez, 2005). Sin embargo, en ocasiones, estos trabajos no clarifican los aspectos de la implicación parental que se ven afectados por el capital cultural. Consideramos que es importante desentrañar esta relación y estudiar el concepto de implicación parental de una forma amplia. Se trata de evitar que se invisibilicen las 
prácticas y estrategias de implicación que ponen en marcha las familias y madres con bajos capitales culturales, como ocurre con gran parte de las familias inmigrantes de origen marroquí.

En este sentido, autores como Espitia y Montes (2009) han subrayado que también las familias con niveles socioeconómicos bajos atribuyen gran importancia a la educación, buscando mejoras en las condiciones de vida y el estatus social. Esto es especialmente cierto entre una buena parte de las familias inmigradas, que tienen como objetivo la mejora social de sus hijas e hijos (Aparicio y Tornos, 2006; Portes et al., 2011; Cebolla et al. 2015). Lo que resulta en grandes esperanzas en la escuela como instrumento que posibilitará estas metas.

De manera que, consideramos que las expectativas y metas educativas de las familias están relacionadas con las estrategias de implicación parental que desarroIlan las madres marroquíes de origen inmigrante. Las estrategias pueden orientarse hacia diferentes aspectos del proceso de aprendizaje como son la motivación, la organización del tiempo, la supervisión, las interacciones y los hábitos de estudio. Pero también a las formas de intercambio de información con la escuela, tal como son las entrevistas con los profesores, la revisión de cuadernos y agendas, las reuniones, etc. No obstante, aunque se estima que las expectativas tienen una alta relación con el rendimiento escolar, es fundamental comprender que se trata de una relación mediada a través de su efecto en variables subjetivas de los individuos, como son la motivación y la autoconfianza (González-Pineda, 2003).

Por tanto, el objetivo de este artículo no es otro que visibilizar las formas y estrategias que las madres marroquíes ponen en funcionamiento para implicarse en la educación de sus hijas e hijos. Al mismo tiempo que se estudia la relación entre implicación parental y los factores sociofamiliares y motivacionales.

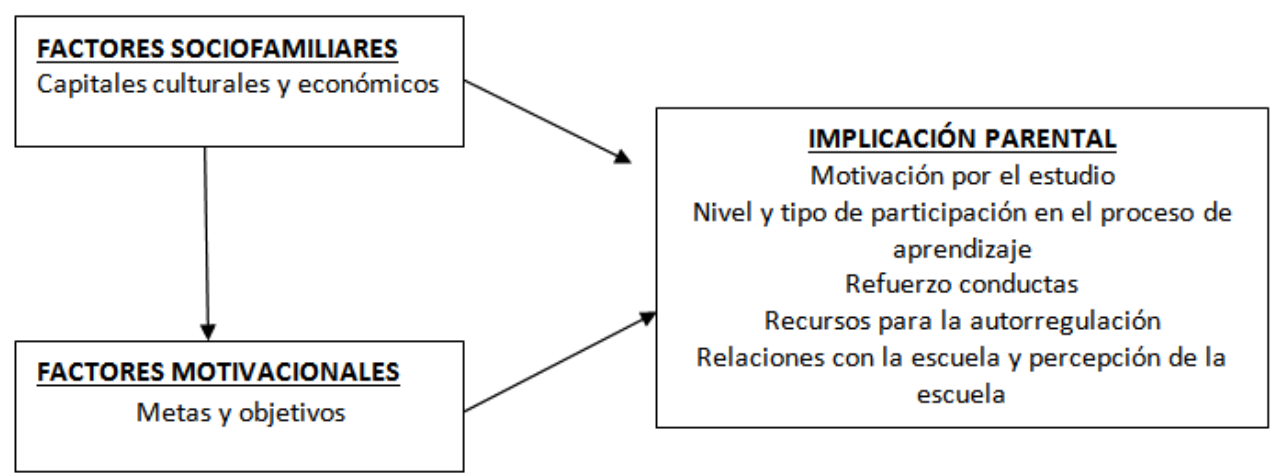

Figura 1. Modelo de análisis

\section{Metodología}

En esta investigación preliminar se ha utilizado una metodología cualitativa basada en el estudio de caso. Hemos trabajado con catorce testimonios, todos ellos de madres marroquíes con hijas e hijos nacidos en España o que Ilegaron a edades 
muy tempranas. Centramos nuestro foco de atención en este perfil al considerar que se trata de un caso diferente al de madres que emigran con hijos en edad escolar, y que se incorporan al sistema educativo español en cursos avanzados de la educación primaria o secundaria.

Las variables de homogeneidad utilizadas para el diseño de la muestra fueron:

1. Mujeres inmigrantes marroquíes.

2. Con al menos tres años de residencia en Cataluña.

3. Que residieran en la ciudad de Barcelona y área metropolitana.

4. Con hijos escolarizados entre los 5 y 12 años.

Mientras que las variables de heterogeneidad fueron:

1. Nivel de estudios.

2. Género del hijo sobre el que se iban a realizarlas preguntas.

Tabla 1. Perfiles de las mujeres entrevistadas y de sus hijas/hijos

\begin{tabular}{|c|c|c|c|c|c|c|c|}
\hline & $\begin{array}{l}\text { EDAD } \\
\text { MADRE }\end{array}$ & $\begin{array}{l}\text { EDAD } \\
\text { HIJO }\end{array}$ & $\begin{array}{c}\text { SEXO } \\
\text { HIJO/A }\end{array}$ & $\begin{array}{l}\text { CURSO } \\
\text { ESCOLAR } \\
\text { HIJO/A }\end{array}$ & $\begin{array}{c}\mathrm{N}^{\circ} \\
\text { HIJOS }\end{array}$ & $\begin{array}{l}\text { LOCALIDAD } \\
\text { ORIGEN }\end{array}$ & $\begin{array}{l}\text { ESTUDIOS } \\
\text { REALIZADOS }\end{array}$ \\
\hline LATIFA & 39 & 5 & M & P5 & 2 & FEZ & UNIVERSITARIOS \\
\hline MOUNIA & 36 & 5 & $\mathrm{H}$ & P5 & 2 & FEZ & UNIVERSITARIOS \\
\hline SOMAYA & 29 & 10 & $\mathrm{H}$ & $2^{\circ}$ & 2 & TANGER & $\begin{array}{l}\text { SECUNDARIOS } \\
\text { OBLIGATORIOS }\end{array}$ \\
\hline NAOAL & 52 & 7 & $\mathrm{H}$ & $1^{\circ}$ & 4 & NADOR & SIN ESTUDIOS \\
\hline JENEF & 45 & 6 & $\mathrm{H}$ & P5 & 1 & $\mathrm{NC}$ & $\begin{array}{l}\text { SECUNDARIOS } \\
\text { OBLIGATORIOS }\end{array}$ \\
\hline SARA & 41 & 9 & M & $3^{\circ}$ & 3 & $\begin{array}{l}\text { OVEZZANE } \\
\text { MAR }\end{array}$ & $\begin{array}{l}\text { SECUNDARIOS } \\
\text { OBLIGATORIOS }\end{array}$ \\
\hline FOUIZIA & 45 & 9 & M & $4^{\circ}$ & 3 & FEZ & UNIVERSITARIOS \\
\hline HADI & 26 & 7 & $\mathrm{H}$ & $1^{\circ}$ & 2 & $\mathrm{NC}$ & $\begin{array}{l}\text { SECUNDARIOS } \\
\text { SIN FINALIZAR }\end{array}$ \\
\hline GEMMA & 45 & 9 & M & $3^{\circ}$ & 3 & TETUAN & $\begin{array}{c}\text { SECUNDARIOS } \\
\text { OBLIGATORIOS SIN } \\
\text { FINALIZAR }\end{array}$ \\
\hline LAIA & 43 & 10 & $\mathrm{H}$ & $4^{\circ}$ & 2 & TETUAN & UNIVERSITARIOS \\
\hline RAFI & 43 & 9 & M & $4^{\circ}$ & 4 & TETUAN & $\begin{array}{l}\text { SECUNDARIOS SIN } \\
\text { FINALIZAR }\end{array}$ \\
\hline HALIMA & 41 & 12 & $M$ & $1 \mathrm{ESO}$ & 4 & MARRAKECH & $\begin{array}{c}\text { SECUNDARIO } \\
\text { POSTOBLIGATORIOS }\end{array}$ \\
\hline FATIMA & 35 & 11 & M & $6^{\circ}$ & 3 & CASABLANCA & $\begin{array}{c}\text { UNIVERSITARIOS SIN } \\
\text { FINALIZAR }\end{array}$ \\
\hline FATIMALI & 33 & 8 & $M$ & $2^{\circ}$ & 2 & MARRAKECH & PRIMARIOS \\
\hline
\end{tabular}


Para abordar el estudio de las estrategias de implicación parental, se dimensionó este concepto de tal forma que recogiera indicadores de motivación, de nivel y tipo de participación en el proceso de aprendizaje, refuerzo de conductas, insistencia y perseverancia, recursos y medios para la autorregulación y presión hacia el logro.

La técnica de recogida de datos utilizada fue la entrevista dirigida. El cuestionario compuesto por 17 preguntas combinaba preguntas con respuestas múltiples y cerradas propias de la encuesta con otras cuestiones abiertas. Esto permitió recoger indicadores altamente sensibles de nuestras variables de estudio y combinarlos con el análisis de los discursos acerca de las prácticas y creencia educativas.

En cuanto al análisis de la información, se realizó un análisis cualitativo multinivel (ACM) que combinó el análisis cuantitativo y cualitativo. Este tipo de análisis se aplica a investigaciones en las que se recopila información procedente de guiones de entrevistas estructuradas o semiestructuradas. El objetivo fundamental del ACM es sistematizar la información de las entrevistas en profundidad en datos que puedan ser utilizados en procedimientos analíticos cuantitativos (con variables categóricas, algunas de ellas cuantitativas). Se trata de un modelo de reducción de la información cualitativa a datos que, cuando menos, pueden ordenarse en rangos según posean mayor o menor "cantidad" del rasgo del atributo. Es decir, el ACEM permite convertir información cualitativa (que en la mayoría de casos puede ser más o menos nominal) en información cualitativa ordinal (Belzunegui et al., 2012). El resultado final que combina el análisis de respuestas abiertas con indicadores fue la construcción de una matriz de datos en Excel que contiene, por un lado, las unidades de análisis y, por otro, las variables categóricas o atributos de aquellas unidades de análisis. En concreto se recogieron 149 indicadores correspondientes a las siguientes variables:

- Datos sociodemográficos

- Nivel de estudios

- Control y norma en el proceso de socialización familiar

- Nivel y tipo de participación en el aprendizaje

- Refuerzo conductas, insistencia perseverancia en condiciones adversas en el proceso de aprendizaje de las hijas/hijos

- Imagen de la hija/o como estudiante

- Expectativas educativas y sociales

- Tiempo que la madre pasa con la hija/o y actividades que realizan

- Control y sobreprotección ejercida sobre la hija/o

- Comprensión y apoyo a la hija/o

- Castigo
- Nivel de presión hacia el logro

- Orgullo y valoración de la hija/o

- Conocimiento de la hija/o

- Motivación por el aprendizaje trasmitida a la hija/o

- Seguimiento de la rutina escolar de la hija/o

- Valoración de la hija/o como estudiante y expectativas educativas

- Nivel de autorregulación del estudio de la hija/o

- Seguimiento del rendimiento escolar de la hija/o

- Valor otorgado a la educación

- Autopercepción de su papel como educadora

- Recursos educativos en el hogar 
Así, se analizaron tanto los discursos como la frecuencia de aparición de determinadas categorías e indicadores. Al tiempo que pudimos valorar el grado de acuerdo entre nuestras entrevistadas en determinadas actitudes y opiniones. Un ejemplo concreto sería la categoría Modelo de Socialización Familiar. Esta variable de estudio se construye tras el análisis de la información considerando si se encuentran o no determinados indicadores de comunicación, afectividad y control sobre la norma. A partir de esta información cualitativa extraída de las entrevistas, se pudo construir una nueva categoría acerca de la intensidad en el control y en la afectividad.

\begin{tabular}{|c|c|c|c|c|}
\hline & Siempre & $\begin{array}{l}\text { Algunas } \\
\text { veces }\end{array}$ & $\begin{array}{l}\text { Cómo/de } \\
\text { qué tipo }\end{array}$ & Nunca \\
\hline $\begin{array}{l}\text { Establecer reglas específicas para su hijo sobre las tareas del } \\
\text { hogar (ayudar en casa, tener responsabilidades, hábitos de } \\
\text { autonomía, etc.) De qué tipo? } \\
\text { Recoger su ropa, sus zapatos, prepararse la mochila para el día } \\
\text { siguiente y la higiene;Si le toca la ducha, ducharse, pero si no le } \\
\text { toca pues lavarse la cara, los pies, las manos y el sulete. }\end{array}$ & $\mathrm{x}$ & & & \\
\hline $\begin{array}{l}\text { Establezco reglas sobre pelear, no pegar, mentir,etc (De qué } \\
\text { tipo las más frecuentes o las más importantes) } \\
\text { Reglas no, a ver les regaño si se pelean o si mienten pero } \\
\text { establecer reglas para que no lo hagan, no. Suelo hablarcon } \\
\text { ellos. }\end{array}$ & & $\mathrm{x}$ & & \\
\hline $\begin{array}{l}\text { Establezco reglas con mi hijo sobre horarios (hora de } \\
\text { levantarse, ir a dormir) (De qué tipo las más frecuentes o más } \\
\text { importantes) } \\
\text { Tiene horarios fijos sobre la hora de levantarse, cenar, irse a } \\
\text { dormir y hacer deberes. }\end{array}$ & $x$ & & & \\
\hline
\end{tabular}

Figura 2. Pregunta 1 del cuestionario

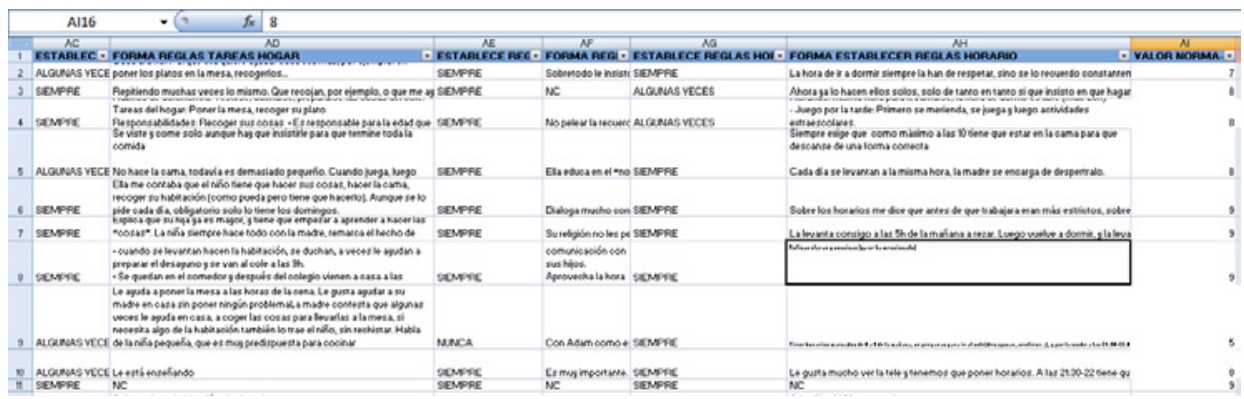

Figura 3. Matriz de datos e indicadores de socialización familiar

El análisis del discurso sobre estas actitudes nos ayudó a comprender las acciones y valores de las madres entrevistadas. Este método también posibilitó considerar en el análisis las variables contextuales de las entrevistadas. Por lo que pudimos estudiar de qué forma los elementos socioeconómicos, demográficos y formativos contextualizan las actitudes y discursos. 


\section{Resultados}

\section{Las madres marroquíes como motor educativo}

Una de las preguntas planteadas en el cuestionario y orientadas a captar indicadores sobre el modelo de socialización familiar fue si: Podrías valorar la importancia que cada una de estas personas tiene en la educación de tu hijo/a (1 era el valor inferior y 5 el valor máximo): Maestro/a; Madre, Padre, Otros (Pregunta 7). Las madres se auto-identificaron como el agente educativo más importante (14 puntos de valoración), seguidos del profesorado (10 puntos), el padre (9 puntos) y otros (7 puntos). Luego interpretamos un alto nivel de compromiso con la educación y un rol muy posicionado en el ámbito familiar respecto a las tareas de crianza. La constatación de este hecho se explica por el modelo de socialización familiar prevalente y la división de roles entre hombres y mujeres.

En nuestro estudio, se examinaron los modelos de socialización familiar a partir del grado de control de normas y la sobreprotección que las madres marroquíes ejercían sobre sus pequeños. Acerca de la sobreprotección, las entrevistadas reconocieron practicar una alta vigilancia sobre las actividades que realizaban sus hijas e hijos. También sobre sus amistades, acerca de dónde se encontraban en cada momento, sus opiniones, decisiones, etc. Lo que tiene sentido si consideramos la edad de las hijas e hijos, entre cinco y doce años ${ }^{2}$.

De igual forma, la totalidad de las familias consultadas reconocieron tener reglas referidas a las actividades del hogar sobre las que las hijas e hijos debían responsabilizarse (hacerse la cama, ayudar a poner y quitar la mesa, vestirse solo, recoger sus cosas...). Además de pelear o mentir o pegar y sobre los horarios. En este sentido no se hallaron diferencias entre niñas y niños ${ }^{3}$.

Acerca de grado de control y norma ejercidos podemos afirmar que este era alto: seis madres puntuaron valores de 6 (siendo este el máximo valor); 5 madres valores de 5 y 2 valores de 3 (el valor mínimo era 3$)^{4}$.

Dentro de las normas familiares, la aplicación de los horarios para levantarse y acostarse era una regla muy observada por las madres entrevistadas. Todas ellas se encontraban en casa por la mañana y en el momento en que los hijos se iban a dormir ${ }^{5}$.

2. En cinco casos se hallaron valores de protección de 12 puntos (valor máximo) y el resto entre 8 y 11 puntos, todos ellos valores por encima del valor medio. Estos valores se calcularon atribuyendo una puntuación a las preguntas planteadas en el cuestionario (multipregunta): Controlamos y nos preocupamos excesivamente por nuestro hijo; Hay muchas cosas que por miedo no le dejamos hacer; Conocemos y controlamos sus amistades. Totalmente de acuerdo (4), Bastante de Acuerdo (3), No muy de acuerdo (2) Nada de acuerdo (1).

3. 10 Familias reconocieron que "Siempre" establecían reglas sobre las tareas del hogar; 12 que "Siempre" establecían reglas sobre pelear, pegar, mentir; 11 que "Siempre establecían reglas sobre los horarios".

4. Los valores se han calculado atribuyendo un valor a la frecuencia con que las entrevistadas reconocieron en la pregunta 1 del cuestionario, llevar a cabo acciones de normativización y control (Siempre=2; Algunas veces $=1 ;$ Nunca $=0$ ).

5. Debemos considerar que seis de las entrevistadas no trabajaban, dos de ellas lo hacían intermitentemente, y entre las que sí trabajaban fuera del hogar, 2 estaban ocupadas en trabajos informales (cuidado de niños, limpieza). Con la excepción de dos casos, el resto eran mujeres activas perjudicadas por el desempleo. 
Por otra parte, gracias a la pregunta 2 del cuestionario, se identificó también un alto nivel de comunicación entre las hijas e hijos y las madres. Casi la totalidad de las madres entrevistadas ${ }^{6}$ reconocieron estar al tanto de los logros escolares y problemas relacionados con la escuela a través de la comunicación. Las madres preguntaban a diario a los pequeños cómo había ido en el colegio o bien la iniciativa la tomaban los niños a la hora de relatarles qué había pasado en la escuela:

La hija le explica las cosas que le pasan en la escuela y fuera de ella. También la madre asiste a los partidos de básquet con ella. Cada semana entra en la aplicación de la escuela por internet donde conoce lo que sucede semanalmente a través de su profesor. Ahí la madre puede ver si asiste a las clases, como va en las asignaturas, si hace los deberes, si se porta bien, etc. A demás concierta entrevistas con su tutor cada dos por tres (Fátima, Estudios Universitarios sin finalizar, con hija de 11 años)...

Igualmente los niveles de compresión y apoyo de las madres dieron resultados elevados, lo que se recogió a partir de los indicadores Respetar Opiniones, Comunicación, Apoyo y Amor (pregunta 9). Las catorce madres reconocieron estar Muy de acuerdo en que "Demuestran frecuentemente apoyo y amor" y que "La comunicación en casa es fundamental y hablamos de nuestras cosas con frecuencia". En menor medida se reconoció estar muy de acuerdo con la afirmación "Respetamos sus opiniones, decisiones, amistades, etc.", lo que puede estar relacionado con la temprana edad de las hijas e hijos ${ }^{7}$. Ante los problemas de mala conducta, casi todas (11 de las madres) se mostraron de acuerdo en recurrir al diálogo y sólo 3 al castigo y la amenaza o regaño.

Yo normalmente... yo me gusta hablar con ella... su padre me dice: "no tienes [que hablar], [tienes] que castigar". Y yo no, es el contrario, me gusta mucho hablar. Tengo que hablar con ella primero. Y si después la otra vez ella hacer más, luego yo castigo a ella. Si ella no lo respeta eso sí se puede castigar. Por ejemplo no mirar la tele y no salir al parque (Sara, Estudios secundarios y con hija de 9 años)

\section{Las estrategias de implicación parental de las madres marroquíes}

Se ha comprobado que la crianza y la socialización son responsabilidad que las madres marroquíes se toman muy en serio. Lo que también abarca la faceta escolar de sus hijas e hijos.

Así, al observar las conductas hacia el aprendizaje de las madres marroquíes, encontramos elevados valores de implicación y apoyo. Para medir esta variable, se planteó la pregunta 11 del cuestionario: Sería tan amable de responder con qué frecuencia... Sigue sus tareas escolares diarias; Comprende las materias que está estudiando; Revisa tareas escolares/agenda; Le programa sus tareas; Le gestiona el tiempo dedicado a tareas y estudio; Evalúa lo que está yendo mal; Le aporta soluciones, le asesora sobre a lo que puede mejorarse en sus estudios; Tiene relación con el tutor/ profesorado; Le motiva y trasmite interés por el estudio ${ }^{8}$.

6. Once de catorce testimonios.

7. 4 casos Muy de acuerdo; 8 bastante de acuerdo.

8. Los valores atribuidos a las respuestas a partir de los que se calculó el valor de intensidad son Siempre $=3$, Algunas veces $=3$; Pocas veces $=1$; Nunca $=0$ El valor máximo es 27 ( 9 preguntas) y el mínimo 0 . 
Como puede apreciarse en la Figura 4, en todos los casos se hallaron valores de implicación por encima del valor medio $(13,5)$.

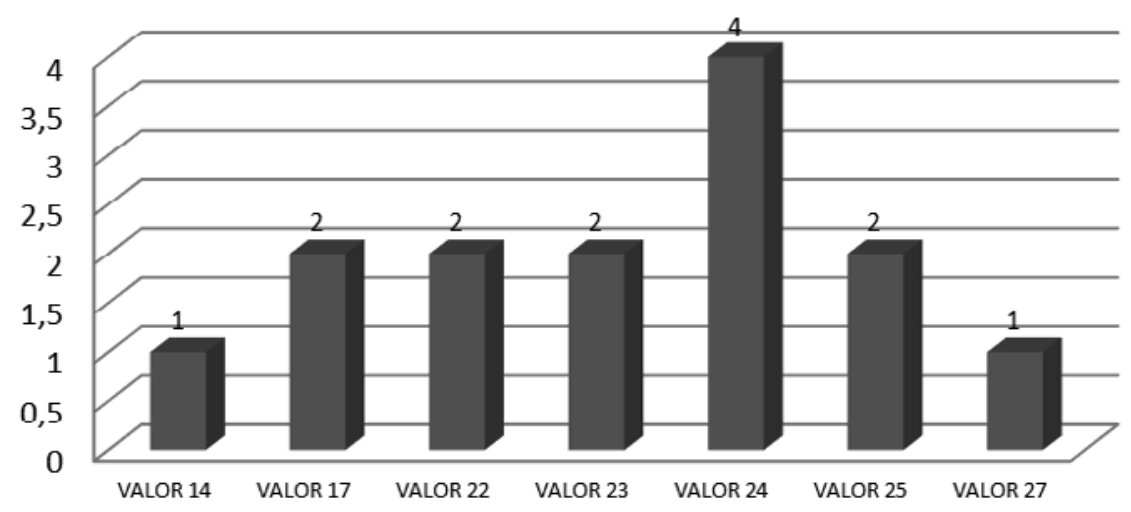

Figura 4. Intensidad del apoyo y ayuda de las madres.

Número de respuestas $(\mathrm{N}=14)$

No obstante, y este es un aspecto relevante de nuestro análisis, se han reconocido divergencias acerca del tipo de actividad de implicación. Tal y como se observa en la Figura 5, todas las madres marroquíes consultadas admitieron altos niveles de implicación al motivar a sus hijas e hijos, en las relaciones con el tutor y en el seguimiento y revisión de tareas. Sin embargo se aprecia cómo decae la implicación en actividades tales como programar tareas, evaluar lo que anda mal y gestionar el tiempo.

MOTIVA, INTERÉS POR ESTUDIO

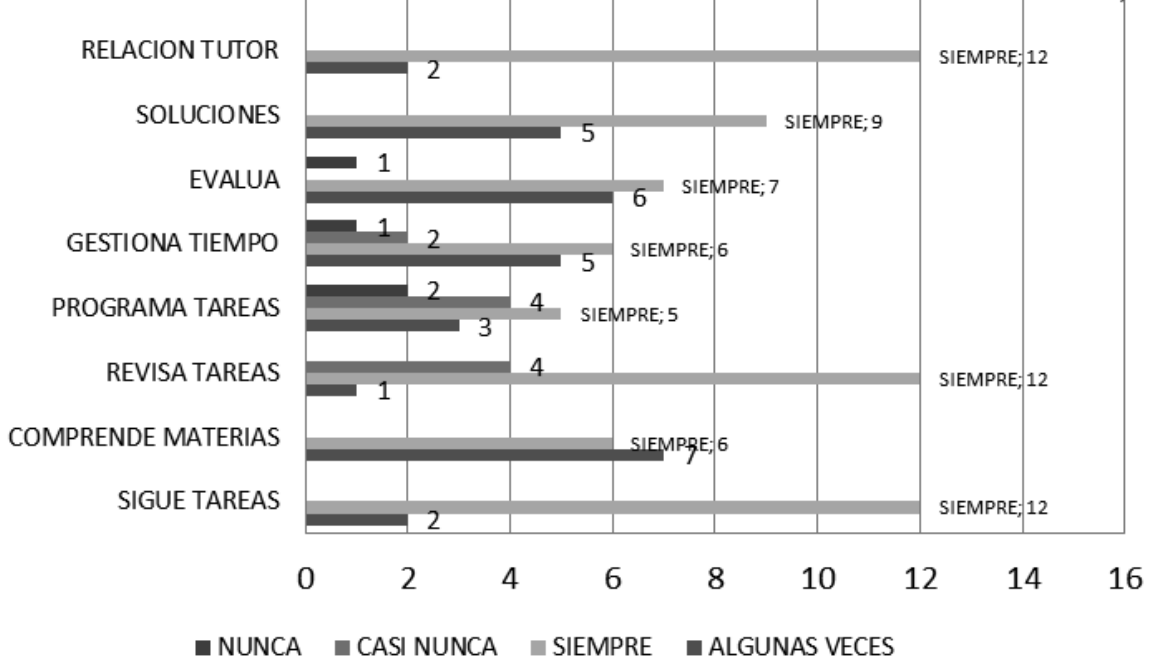

Figura 5. Apoyo de las madres marroquíes en los estudios. Números de casos 
Por otra parte, y en términos generales, las principales dificultades que reconocieron las madres marroquíes para poder involucrarse en los estudios de sus hijas e hijos fueron:

- El idioma, también entre las madres con estudios universitarios (8 casos lo reconocieron $)^{9}$ :

- El contenido curricular (5 casos lo reconocieron) ${ }^{10}$ :

Cada vez van creciendo más y van avanzando y no les puedo ayudar y me cuesta. En el idioma puedo ayudarla pero hay vocabulario que me pregunta y no lo sé pero lo buscamos en el diccionario. Su padre ayuda poco en el catalán pero algo de matemáticas sabe. (Latifa, estudios universitarios, con hija de 5 años).

Estas dificultades concuerdan con otros estudios sobre madres marroquíes como el de Jordi Garreta (1994). De hecho la mitad de las madres entrevistadas declararon que sólo "A veces" comprendían las materias curriculares de sus hijas e hijos. Con todo, resulta notorio cómo las madres acostumbraban a buscar estrategias de acompañamiento que pasaban por "apuntarse a clases de catalán" (Sara), buscar ayuda en internet como es utilizar el traductor de Google (Fouzia y Fátima), pedir ayuda a otros hijos (Naoal; Gemma; Halima), buscar ayuda entre otras madres de la clase (Somaya) o acudir a la maestra (Rafi). En otras palabras, desarrollaban modelos de implicación basados en la facilitación de ayuda.

[La madre] Tiene dificultades a la hora de ayudarle por el hecho de no comprender bien el idioma y por el hecho de no tener estudios. Le ayudan siempre sus hermanos. Está aprendiendo a leer. Trae libros del colegio pero no está muy interesado en ellos. Su hermana mayor, que entiende el español es quien le ayuda con la lectura y los deberes. (Naoal, sin estudios y con un hijo de 7 años).

\section{Actitudes ante la escuela de las madres marroquíes inmigrantes}

Las altas expectativas de la mayoría de las madres y la seguridad que parece inspirarles el sistema educativo catalán y español nos ayuda a comprender las posturas de adhesión y adaptación que estas familias tienen frente a la escuela.

La frecuencia de las reuniones formales e informales con el tutor o tutora de sus hijas e hijos es un indicador no sólo de la implicación de estas madres, sino, también, de la relación que mantienen con el colegio. Así, se hizo visible que la mayoría de las madres entrevistadas (once de ellas) mantenían una comunicación muy fluida con los tutores de sus hijas e hijos a través de conversaciones informales a la entrada o salida del aula, de las reuniones formales y periódicas y otros métodos de contacto como la agenda, el correo electrónico o el teléfono. Madres como Halima (Estudios secundarios postobligatorios, con hija de 12 años), nos explicaron que cada semana

9. Latifa con estudios universitarios; Mouina con estudios universitarios; Naoal sin estudios; Sara con estudios secundarios obligatorios; Fouizia con estudios universitarios; Gemma con estudios secundarios obligatorios sin terminar; Halima con estudios post-obligatorios; Fatimali con estudios primarios.

10. Latifa con estudios universitarios, con una hija de 5 años; Naoal sin estudios un niño de 7 años; Halima con estudios post-obligatorios una niña de 12 años; Fatima con estudios universitarios sin finalizar con un niño de 11 años; Fatimali con estudios primarios y una niña de 8 años. 
se comunicaba con el maestro a través de un recurso on-line que proporcionaba la misma escuela y además cada dos meses solicitaba tutorías para hablar cara a cara con el tutora/a acerca de la evolución de su hija. Observamos, también, que fueron muchas las madres que recurrían a más horas de entrevistas de las habituales para pedir la opinión y ayuda del profesorado. Sólo tres casos consideraron que las entrevistas y reuniones propuestas desde la escuela eran suficientes, o que únicamente las solicitaban cuando lo consideran estrictamente necesario con el fin de solucionar problemas graves de conducta o de resultados académicos (Gemma, Rafi, Fatima).

Por otra parte, y a pesar de que la mayoría de las mujeres entrevistadas consideraban la escuela como un instrumento de mejora social, las madres marroquíes reconocieron en la educación objetivos mucho menos instrumentales de los que cabría esperar, dado el predominio de objetivos educativos de mejora social. De este modo, la mayoría de las madres, manifestaron estar muy o bastante de acuerdo con las afirmaciones planteadas en la pregunta 16 del cuestionario acerca de que "Estudiar es importante para ser un buen profesional" (12 casos) y "Estudiar es importante para ser feliz" (10). Al contrario, se reconoció estar muy poco de acuerdo con la afirmación de que "Estudiar es importante para ganar dinero" (12 casos indicaron estar Muy poco de acuerdo o Nada de acuerdo).

\section{Discusión Y CONCLUSIONES}

Algunas investigaciones han revelado ciertos prejuicios en el ámbito escolar acerca de las mujeres madres marroquíes. Estas sospechas sostienen que las mujeres madres marroquíes están poco interesadas en la educación de sus hijas e hijos, lo que se traduce en una baja implicación en las cuestiones escolares (Alcalde, 2007; Tovías, 2002; Carrasco et al., 2012). El escaso interés explicaría, según estos argumentos, los peores resultados académicos de estos estudiantes. Empero, muy al contrario, los estudios sobre mujeres y madres marroquíes, donde se ubica nuestra investigación, desmienten este prejuicio. Tal y como se ha comprobado aquí también, la implicación en los estudios de las mujeres madres marroquíes, es alta, lo que, a su vez, se traduce en un elevado interés, seguimiento y apoyo en las experiencias escolares de hijas e hijos. Estos hallazgos resultan razonables si consideramos que las mujeres madres marroquíes ejercen un rol central en la educación y crianza de sus hijas e hijos (Llorent y Terrón, 2013; Terrón, 2008). Estudios como el de Naima Ejbari (2013) apoyan nuestros resultados donde se demuestra que son las madres las que participan más en la actividad escolar de las hijas e hijos, a pesar de las dificultades económicas, lingüísticas o de aprendizaje que puedan tener.

Podemos afirmar que entre las familias consultadas prevalecen modelos de socialización familiar caracterizados por una alta afectividad y comunicación y un alto control de la norma. Lo que se reconocen como modelos contractuales o conductivistas o autorizativos. Está demostrado que estas formas de relación tan basadas en el afecto y la comunicación facilita la implicación parental.

Por otro lado, las madres marroquíes desarrollan estrategias de acompañamiento basándose en los recursos sociales y educativos de su entorno para superar las dificultades con el idioma vehicular de la escuela y con el contenido curricular. Lo 
que indica lo importante que resulta que estas mujeres y madres posean y accedan a recursos tanto sociales (red social fuera y dentro del colegio, relación con el profesorado) como educativos (materiales educativos, bibliotecas, etc.) que les faciliten el acompañamiento escolar de sus hijas e hijos. En este sentido las relaciones con el profesorado se han manifestado como algo fundamental. Estudios como el de Rosa Aparicio y Andrés Tornos (2006) indican que las familias de origen inmigrante tienden a tener una mejor valoración de la escuela y del profesorado que las familias autóctonas. También nuestros datos indican claramente que las madres marroquíes valoraban la relación con el profesorado como algo importante y necesario. De hecho, desarrollan un contacto frecuente con el profesorado y no se observaron diferencias destacables en función a los capitales culturales. En este sentido, hay que considerar que en todas las familias marroquíes estudiadas, las hijas e hijos habían nacido en España o habían llegado con apenas unos meses de vida y que sus madres procedían en su mayoría de entornos urbanos. Creemos que el tiempo de relación entre las familias y la institución escolar es un factor que probablemente explique las diferentes relaciones que mantienen otro tipo de madres inmigrantes marroquíes con la escuela.

Ahora bien, aunque nuestro estudio demuestra que las madres marroquíes están altamente implicadas en la educación de sus hijas e hijas debido a sus expectativas educativas y sociales de mejora, se ha identificado un posible efecto negativo relacionado con los bajos capitales culturales que puede afectar a un bajo rendimiento escolar. Éste tiene que ver con los estilos motivacionales orientados al rendimiento, las bajas expectativas sociales y la no trasmisión de pautas de autorregulación.

Entre las familias entrevistadas, cómo ocurre entre otras muchas familias inmigrantes, las metas u objetivos educativos están imbricados con las expectativas sociales. Por lo que podemos decir que la motivación está fuertemente vinculada a lo que se reconoce como objetivos de rendimiento. En este sentido, se identifican en la observación que existen diversos objetivos educativos entre las madres consultadas, muchos de ellos de carácter más afectivo que instrumental. Éste nos parece un descubrimiento relevante sobre el que indagar ya que indica que si bien la meta educativa y social entre estas familias es mejorar, el objetivo no es el "éxito social", o no al menos como lo entienden las clases medias y medias altas. Como indica Martín Criado (2010), la percepción de éxito depende del punto de partida, es decir, de la posición social de origen. Por lo que estimamos que estos datos obligan a matizar la afirmación de estudios como el de Aparicio y Tornos que afirman que la mayoría de las familias inmigrantes tienen expectativas más altas que las de los autóctonos. En realidad, lo que el análisis cualitativo nos está sugiriendo es que tienen expectativas de mejora. Por lo que aquello que valoran no es tanto el punto de llegada sino el recorrido. Cabe preguntarse hasta qué punto esta expectativa puede influir en el rendimiento escolar. Dado que conocemos que las expectativas ejercen una influencia sobre la motivación hacia el estudio a través de su impacto en la autoimagen como estudiante (González-Pineda, 2003; y Zimmerman y Martínez-Pons, 1988). En definitiva, nuestro trabajo sugiere que la forma en que se configuran las metas sociales y sus efectos en la motivación explican mejor los estilos de implicación parental y sus efectos en las experiencias escolares de los descendientes

A su vez las metas y objetivos afectan a las estrategias que utilizan las madres para motivar a sus hijas e hijos. La forma predominante de motivación hacia el estudio 
hallada en nuestra muestra fue a través del refuerzo de la conducta con premios. Con lo que se motiva a las niñas y niños hacia el logro y la experimentación de orgullo y satisfacción, lo que se conoce como metas de rendimiento. Este tipo de metas correlaciona menos con el rendimiento escolar. En muy pocas ocasiones se hallaron estrategias de motivación orientadas al aprendizaje y en consecuencia a mejorar la propia competencia e interés, que sí muestran una alta correlación con el rendimiento. Las metas de aprendizaje están influidas por los capitales culturales de las madres. No obstante, el tamaño de nuestra muestra no nos ha permitido evaluar bien la relación entre estilos motivacionales y capitales culturales.

Finalmente, uno de los hallazgos más importantes del estudio es el de reconocer que las madres marroquíes, a pesar de su alta implicación, presentan una debilidad en aquellos aspectos relativos a la autorregulación del estudio. Sabemos por la investigación acumulada (Martínez-Pons, 1996; Zimmerman y Martínez-Pons, 1996), que las conductas de autorregulación son fundamentales para el desarrollo escolar. Lo que se traduce en aprender a formular y asumir metas concretas, próximas y realistas; planificar, observar y evaluar la actuación; evaluar la eficacia de las acciones para conseguir las metas y reaccionar y ajustar las acciones. Los estudios especializados (Quaresma, 2013; Kellerhals y Montandon, 1991; Torres y Silva, 1998) indican que entre las familias de clases sociales altas, se valora mucho más el desarrollo de la autorregulación y que es una prioridad entre las familias con altos capitales educativos. El autodominio es considerado como una meta para las clases dominantes ya que se asume que esto potencia el éxito escolar (Lautrey, 1980). Así, las clases altas se implican para la autonomía, la responsabilidad y el autocontrol. Mientras que en los grupos analizados de madres inmigrantes de clase obrera se centran en el seguimiento escolar y en la motivación orientada al rendimiento.

\section{REFERENCIAS BIBLIOGRÁFICAS}

Alcalde, R. (2007). Igualar en la diversidad: La atención educativa del alumnado de origen extranjero en los centros de educación primaria de Cataluña y las Islas Baleares. (Tesis Doctoral). Fac. Ciències Polítiques i Sociologia. Universitat Autònoma de Barcelona.

Alcalde, R. y Hernández, R. (2012). La recompensa del esfuerzo: Estructuras de implicación educativa de las familias inmigrantes dominicanas y efectos en la motivación y autoregulación del estudio como claves del éxito. (Informe de investigación.). Barcelona: Institut d'Estudis Catalans.

Aparicio, R. y Tornos, A. (2006). Hijos de inmigrantes que se hacen adultos: Marroquíes, dominicanos, peruanos. Madrid: Ministerio de Trabajo y Asuntos Sociales.

Appelbaum, P. (2002). Multicultural and Diversity Education. A Reference Book. California: ABC Clio.

Belzunegui, A., Brunet, I. y Pastor, I. (2012). El diseño del análisis cualitativo multinivel: Una aplicación práctica para el análisis de entrevistas. Empiria. Revista de Metodología de Ciencias Sociales, 23,15-44.

Carbonell, F. (2000). Educació i immigració: Els reptes educatius de la diversitat cultural i l'exclusió social. Barcelona: Mediterrània. 
Carrasco, S., Pàmies, J. y Ponferrad A. M. (2012). Fronteras visibles y barreras ocultas. Aproximación comparativa a la experiencia escolar del alumnado marroquí en Cataluña y mexicano en California. Migraciones, 29, 31-60.

Cebolla, H. y Martínez de Lizarrondo A. (2015). Las expectativas educativas de la población inmigrante en Navarra. ¿Optimismo inmigrante o efectos de escuela? Revista internacional de sociología, 73,1. DOI: http://doi.org/10.3989/ris.2013.02.22.

Collet, J. y Tort. A. (Coords.) (2011). Escola, famílies i èxit. Barcelona: Fundació Jaume Bofill.

Ejbari, N. (2013). El rol de la familia en la educación de los hijos antes y después de la migración. (Tesis doctoral). Universidad de Almería. Almería.

Elosegui, K. y Etxeberría, F. (2010). Integración del alumnado inmigrante: obstáculos y propuestas. Revista Española de Educación Comparada, 16, 235-263.

Espitia, R. y Montes, M. (2009) Influencia de la familia en el proceso educativo de los menores en el barrio de Costa Azul de Sincelejo (Colombia). Investigación y desarrollo, 17(1), 85-105.

Garreta, J. (1994). Expectativas educativas y sociales de las familias inmigrantes Papers: revista de sociología, 43, 115-122.

González Cabanach, R., Valle Arias, A., Núñez Pérez, J. C. y González-Pineda, J. A. (1996). Una aproximación teórica al concepto de metas académicas y su relación con las motivación. Psicothema, 8(1), 45-61.

González-Pineda, J. A. et al. (2003). Adaptabilidad y cohesión familiar, implicación parental en conductas autorregulatorias, autoconcepto del estudiante y rendimiento académico. Psicothema, 15(3), 471-477.

Kellerhalls, J. C. y Montandon, C. (1991). Les Stratégies éducatives des familles : milieu social, dynamique familiale et éducation des pré-adolescents. Lausanne, Delachaux et Niestlé.

Lautrey, J. (1980). Classe sociale, milieu familial, intelligence. Paris: Presses Universitaires de France.

Llorent, V. y Terron, T. (2013). La inmigración marroquí en España: Género y educación. Estudios sobre educación, 23, 37-59.

Marjoribanks, K. (1979). Family environments. En Walberg, E. J. (Ed.). Educational environmenís and effects: Evaluation, policv and productivitv. Berkeley, California: McCutchan.

Martín Criado, E. (2010). La escuela sin funciones; Crítica de la sociología de la educación crítica. Bellaterra: Bellaterra.

Martínez González, R. A. y Álvarez Blanco, L. (2005). Fracaso y abandono escolar en Educación Secundaria Obligatoria: Implicación de la familia y los centros escolares. Aula Abierta, 85, 127-146

Martínez-Pons, M. (1996). Test of a model of parental inducement of academic selfregulation. The Journal of Experimental Education, 64, 213-227.

Musitu, G. y García, J. F. (2001). ESPA29. Escala de Socialización Parental en la Adolescencia. Madrid: TEA Ediciones.

Padilla, A. y González, R. (2001). Academic Performance of Immigrant and U.S.-Born Mexican Heritage Students: Effects of Schooling in Mexico and Bilingual/English Language Instructions. American Educational Research Journal, 38(3), 3-41. 
Portes, A., Aparicio, R., Haller, W. y Vickstrom, E. (2011). Progresar en Madrid: aspiraciones y expectativas de la segunda generación en España. Revista Española de Investigaciones Sociológicas, 134, 55-86.

Quaresma, M. L. (2013). Socialización familiar y formación holística: análisis de las prácticas educativas de las clases dominantes. Revista de la Asociación de Sociología de la Educación, 6(3), 491-503.

Ruiz de Miguel, C. (2001). Factores familiares vinculados al bajo rendimiento. Revista Complutense de Educación, 12(1), 81-113.

Suárez-Orozco, C. y Suárez-Orozco, M. (2008). Històries d'immigració: La comprensió dels patrons de rendiment escolar dels joves immigrants nouvingut. Barcelona: Fundació Jaume Bofill.

Torres, A. y Silva, F. (1998). Guarda das crianças e divisão do trabalho entre homens e mulheres. Sociologia, Problemas e Práticas, 28, 9-65.

Terrón, T. (2008). La perspectiva de las madres marroquíes sobre la educación de sus hijos e hijas en Huelva y en las zonas de origen. Revista Española de Educación Comparada, 14, 3-135.

Tovías Wertheimer, S. (2002). Experiencias de intervención y formación del profesorado en contextos de desigualdad y multiculturalidad en Cataluña. (Tesis Doctoral). Facultat de Lletres. Universitat Autònoma de Barcelona.

Zimmerman, B. J. y Martínez-Pons, M. (1988). Construct validation of a strategy model of student self-regulated learning. Journal of Educational Psychology, 80, 284-290. 